\title{
TRANSFORMATIONS FROM A LINEAR SPACE WITH WEAK TOPOLOGY
}

\author{
ERNEST MICHAEL
}

1. Introduction. One of the important problems in the theory of topological linear spaces is to determine the space $\mathcal{L}(E, G)$ of continuous linear transformations from a topological linear space ${ }^{1} E$ to a topological linear space $G$. In this paper we study the case where the topology on $E$ is the weak topology generated by a separating family $K$ of linear transformations ${ }^{2} T: E \rightarrow F_{T}$ from $E$ to topological linear spaces $F_{T}\left(F_{T}\right.$ may be different for different $\left.T \in K\right)$, and where $G$ is a normed linear space. ${ }^{1}$

If $T \in K$, and $S \in \mathcal{L}\left(F_{T}, G\right)$, then clearly $S T \in \mathcal{L}(E, G)$, and hence the set $L$ of finite linear combinations of such $S T$ is a subspace of $\mathcal{L}(E, G)$. The purpose of this paper is to determine under what conditions the system $\left\{E,\left\{F_{T}\right\}, K, G\right\}$ has the following property:

$$
L=\mathcal{L}(E, G) \text {. }
$$

The validity of (1) has hitherto been established in the following two cases:

I. All the $F_{T}$, as well as $G$, are the field of scalars [1, Theorem 1].

II. All the $F_{T}$ are a Hilbert space $H, E$ is the space of bounded operators on $H, K=\left\{T_{x}\right\}_{x \in H}$, where $T_{x}: E \rightarrow H$ is defined by " $T_{x}(A)=A(x)$," and $G$ is the field of scalars [2, Theorem 4c].

In Theorem 1 of $\$ 2$, we shall give a very general sufficient condition for the validity of (1). In Proposition 1 we show that the validity of (1) for a given system implies its validity for certain related systems. In Proposition 2, we show that (1) is valid in a strong sense for an important particular system. In $\$ 3$ we give some examples of systems which satisfy the condition of Theorem 1; Case I above is a special case of Examples 1 or 2 or 3 or 4, while Case II is a special case of Examples 4 or 5 . In $\$ 4$, finally, we give some examples of systems which do not have property (1), and of systems which have property (1) but do not satisfy the condition of Theorem 1.

The letters $E, F_{T}, K, G$, and $L$ will retain the meaning assigned to them above throughout this paper; to describe a particular system,

Received by the editors March 6, 1951 and, in revised form, December 26, 1951.

1 The field of scalars is either the real numbers or the complex numbers.

2 I.e., the weakest topology on $E$ for which all the elements of $K$ are continuous. A sub-base for the neighborhoods of $0 \in E$ for this topology provided by the sets of the form $T^{-1}(U)$, where $T \in K$ and $U$ is a neighborhood of $0 \in F_{T}$. 
we shall merely specify $E,\left\{F_{T}\right\}, K$, and $G$. In many of our examples, all the $F_{T}$ will be equal to a space $F$; to indicate this, we shall simply specify the space $F$.

\section{The theorems.}

Theorem 1. In order that a system $\left\{E,\left\{F_{T}\right\}, K, G\right\}$ have property (1), it is sufficient that it satisfy the following condition:

(2) If $\Im=\left\{T_{1}, \cdots, T_{n}\right\}$ is any finite subset of $K$, and if we define the linear transformations $J: E \rightarrow F_{T_{1}} \times \cdots \times F_{T_{n}}$ by " $J(x)$ $=\left(T_{1}(x), \cdots, T_{n}(x)\right)$," then any continuous linear transformation $V$ from $J(E)$ to $G$ can be extended to a continuous linear transformation $S$ from $F_{T_{1}} \times \cdots \times F_{T_{n}}$ to $G$.

Proof. Let $U \in \mathcal{L}(E, G)$. Then, by the continuity of $U$, and the definition of the (weak!) topology on $E$, there exists a finite subset $\Im=\left\{T_{1}, \cdots, T_{n}\right\}$ of $K$, and for each $i(i=1, \cdots, n)$ a neighborhood $V_{i}$ of 0 in $F_{T_{i}}$, such that

$$
T_{i}(x) \in V_{i}(i=1, \cdots, n) \rightarrow\|U(x)\|<1 .
$$

Now $T_{i}(x)=0(i=1, \cdots, n) \rightarrow T_{i}(\lambda x)=0$ for $(i=1, \cdots, n)$ and any scalar $\lambda \rightarrow|\lambda|\|U(x)\|=\|U(\lambda x)\|=0$ for any $\lambda \rightarrow U(x)=0$. Hence $J(x-y)=0 \rightarrow T_{i}(x-y)=0 \quad(i=1, \cdots, n) \rightarrow U(x-y)=0$. We can therefore unambiguously define a linear transformation $\tilde{U}: J(E) \rightarrow G$ by

$$
\tilde{U}(J(x))=U(x)
$$

Now $\tilde{U}$ is also continuous, since $J(x) \in V_{1} \times \cdots \times V_{n} \rightarrow T_{i}(x) \in V_{i}$ $(i=1, \cdots, n) \rightarrow\|\tilde{U} J(x)\|=\|U(x)\|<1$.

Now suppose that (2) is satisfied. Then $\tilde{U}$ can be extended to a continuous linear transformation $S: F_{T_{1}} \times \cdots \times F_{T_{n}} \rightarrow G$. Let us make the following convention: if $y \in F_{T_{i}}$, then $y^{\prime}$ denotes that element of $F_{T_{1}} \times \cdots \times F_{T_{n}}$ whose $i$ th coordinate is $y$, and all of whose other coordinates are 0 . Now define $S_{i}: F_{T_{i}} \dot{\rightarrow} G$ by " $S_{i}(y)=S\left(y^{\prime}\right)$ "; each $S_{i}$ is clearly a continuous linear transformation, and $S\left(\left(y_{1}, \cdots, y_{n}\right)\right)=S\left(y_{1}^{\prime}+\cdots+y_{n}^{\prime}\right)=S_{1}\left(y_{1}\right)+\cdots+S_{n}\left(y_{n}\right)$. Hence $U(x)=\tilde{U} J(x)=S\left(\left(T_{1}(x), \cdots, T_{n}(x)\right)\right)=S_{1} T_{1}(x)+\cdots+S_{n} T_{n}(x)$ for all $x \in E$. But this implies that $U=S_{1} T_{1}+\cdots+S_{n} T_{n}$, which is what was to be proved. Q.E.D.

The following simple proposition is sometimes quite useful in extending results obtained by means of Theorem 1. Applications of this proposition, for instance to Example 5 of the next section, are left to the reader. 
Proposition 1. If the system $\left\{E,\left\{F_{T}\right\}, K, G\right\}$ has property (1), if $G$ is a Banach space, and if $E^{\prime}$ is a dense subspace of $E$, then $\left\{E^{\prime},\left\{F_{T}\right\}, K^{\prime}, G\right\}$ also has property (1), where $K^{\prime}$ is the family of all $T \mid E^{\prime}$ with $T \in K$.

Proof. Let $U^{\prime} \in \mathcal{L}\left(E^{\prime}, G\right)$. Since $E^{\prime}$ is dense in $E$, and $G$ is complete, we can extend $U^{\prime}$ to a transformation $U \in \mathcal{L}(E, G)$. Since $\left\{E,\left\{F_{T}\right\}, K, G\right\}$ has property (1), we have $U=S_{1} T_{1}+\cdots+S_{n} T_{n}$, where $T_{i} \in K$ and $S_{i} \in \mathcal{L}\left(F_{T_{i}}, G\right)(i=1, \cdots, n)$. But then $U^{\prime}$ $=S_{1} T_{1}^{\prime}+\cdots+S_{n} T_{n}^{\prime}$, where $T_{i}^{\prime}=T_{i} \mid E^{\prime}$ is an element of $K^{\prime}$. Q.E.D.

Consider a locally convex topological linear space with topology $\tau$, and let $\left\{p_{\alpha}\right\}$ be a basic set of continuous pseudonorms for $E$. If $N_{\alpha}=\left\{x \in E \mid p_{\alpha}(x)=0\right\}$, then $N_{\alpha}$ is a subspace of $E$, and $p_{\alpha}(x)=p_{\alpha}(y)$ whenever $x-y \in N_{\alpha}$. Let $F_{\alpha}$ be the linear space $E / N_{\alpha}$, normed by " $\left\|\bar{x}_{\alpha}\right\|=p_{\alpha}(x)$." If $T_{\alpha}$ is the natural mapping of $E$ onto $F_{\alpha}$, it follows from the definitions that $\tau$ is precisely the weak topology on $E$ induced by the set $K$ of all such $T_{\alpha}$. We now have the following proposition:

Proposition 2. If $E,\left\{F_{\alpha}\right\}$, and $K$ are as above, and $G$ is arbitrary, then (1) holds. As a matter of fact, every element of $\mathcal{L}(E, G)$ is of the form $S T_{\alpha}$, where $T_{\alpha} \in K$ and $S \in \mathcal{L}\left(F_{\alpha}, G\right)$.

Proof. If $U \in \mathcal{L}(E, G)$, then there exists an $\alpha$ such that $\|U(x)\|<1$ whenever $p_{\alpha}(x)<1$. Hence $U(x)=0$ whenever $p_{\alpha}(x)=0$, and we may therefore define a linear transformation $S: F_{\alpha} \rightarrow G$ by " $S(\bar{x})=U(x)$." Now clearly $S$ is continuous, and therefore $S \in \mathcal{L}\left(F_{\alpha}, G\right)$; since furthermore $U=S T_{\alpha}$, this proves our proposition. Q.E.D.

Let us now conclude this section with a lemma which we need in Example 2 of $\$ 3$ below; in the proof we shall again use the methods developed in the paragraph preceding Proposition 2.

LEMMA 1. If, for every normed linear space $E$, the normed linear space $G$ has the property that every continuous linear transformation from a subspace of $E$ into $G$ can be extended to a continuous linear transformation from $E$ into $G$, then $G$ has this property for every locally convex topological linear space $E$.

Proof. Suppose that $G$ has the property of the lemma for every normed linear space $E$, and let $E$ be a locally convex topological linear space. Let $M$ be a subspace of $E$, and let $U$ be a continuous linear transformation from $M$ into $G$; we must find a continuous linear transformation from $E$ into $G$ which extends $U$. Since $U$ is continuous, there exists a continuous pseudonorm $p$ on $E$ such that $\|U(x)\|<1$ whenever $p(x)<1$. Now let $F$ be the normed space gen- 
erated by $E$ and $p$ just as in the paragraph preceding Proposition 2, and let $T$ be the natural mapping from $E$ onto $F$. We may now define the linear transformation $S: T(M) \rightarrow G$ by " $S(T(x))=U(x)$ "; clearly $S$ is continuous, and $U=S T$. By assumption, we may now extend $S$ to a continuous linear transformation $\tilde{S}: F \rightarrow G$. But then $\tilde{S} T$ is a continuous linear transformation from $E$ into $G$ which extends $U$, and this proves our lemma. Q.E.D.

3. Examples of systems which satisfy condition (2). In this section, all $F_{T}$ and $F$ are assumed to be locally convex.

EXAMPLE 1. $G$ is the field of scalars. (This follows immediately from the Hahn-Banach theorem.)

The next example generalizes Example 1:

EXAmple 2. $G$ has the binary intersection property for spheres. (This follows from [4, Theorem 1] and Lemma 1.)

EXAmple 3. Each $T \in K$ has finite-dimensional range. $(J(E)$ is then finite-dimensional. Since $F_{T_{1}} \times \cdots \times F_{T_{n}}$ is locally convex, there exists a continuous projection $P$ from $F_{T_{1}} \times \cdots \times F_{T_{n}}$ onto $J(E)$, and $V P$ is the required extension of $V$.)

ExAmple 4. Each $F_{T}$ is a pre-Hilbert space, ${ }^{3}$ and $G$ is a Banach space. $\left(F_{T_{1}} \times \cdots \times F_{T_{n}}\right.$ is a pre-Hilbert space, with completion $H$. Also $V$ can be extended to a continuous linear transformation $\bar{V}$ from $M$, the closure of $J(E)$ in $H$. If $P$ is the projection from $H$ onto $M$, then the restriction of $\bar{V} P$ to $F_{T_{1}} \times \cdots \times F_{T_{n}}$ is the required extension of $V$. )

Example 5. All the $F_{T}$ are equal to some $F, E$ is an algebraically dense $^{4}$ space of linear transformations from a linear space $D$ to $F$, and $K=\left\{T_{x}\right\}_{x \in D}$, where $T_{x}: E \rightarrow F$ is defined by " $T_{x}(A)=A(x)$." (For in this case, $J(E)$ is all of $F_{T_{1}} \times \cdots \times F_{T_{n}}$, and hence no extension is necessary.)

In Example 5, the topology on $E$ is the familiar strong topology on operators. In view of this example, it becomes interesting to know under what conditions the space $E$ of linear transformations is algebraically dense. ${ }^{4}$ We now give three such conditions: the first two are well known, and the last one, which is a slight improvement on [7, Theorem 4.1], is proved in J. Dieudonné's review of [7].

(1) $D$ is locally convex, and $E$ contains all continuous linear trans

I.e., a normed linear space whose completion is a Hilbert space.

- A ring $R$ of linear transformations from a linear space $M$ into a linear space $N$ is $n$-transitive if, whenever $x_{1}, \cdots, x_{n}$ are linearly independent in $M$, and $y_{1}, \cdots, y_{n}$ are arbitrary in $N$, there exists a $T \in R$ such that $T\left(x_{i}\right)=y_{i}(i=1, \cdots, n)$. If $R$ is $n$-transitive for all $n=1,2, \cdots$, then $R$ is dense. 
formations from $D$ to $F$ with finite-dimensional range. (This follows from the Hahn-Banach theorem.)

(2) $D=F$, and $E$ is a 2-transitive $e^{4}$ algebra of operators. (See [3, Theorems 7 and 6].)

(3) $F$ is a complex normed space, $D=F$, and $E$ is a 1-transitive algebra of continuous operators. (The proof is almost identical with that of [6, Theorem 3.2].)

4. Some counter-examples. We conclude by giving some examples of systems which do not have property (1) (Ex. 6 and Ex. 8), and of systems which have property (1) but do not satisfy condition (2) of Theorem 1 (Ex. 7 and Ex. 9).

In Ex. 6 and Ex. 7 below, $B$ denotes a Banach space which contains a subspace onto which there is no continuous projection from $B$, and $M$ denotes that subspace. (See, for instance, [5, Theorem 7.5].) Furthermore, $\alpha$ (resp. $\beta$ ) denotes the identity map of $M$ into $B$ (resp. $M$ ).

Example 6. $E=G=M, F=B$, and $K=\{\alpha\}$. Then (1) does not hold, since $\beta \in \mathcal{L}(E, G)$ but $\beta \notin L$.

EXAMPLE 7. $E=G=M, F_{\alpha}=B, F_{\beta}=M, K=\{\alpha, \beta\}$. Then (1) clearly holds. But (2) does not hold, which is seen by taking $\Im=\{\alpha\}, V=\beta$.

When examining the above examples, we notice that the system in Ex. 6 does not satisfy condition (a) below, while the one in Ex. 7 satisfies neither (a) nor (b).

(a) Each $T$ is onto $F_{T}$.

(b) All $F_{T}$ are identical with $G$.

That even these conditions are insufficient to guarantee good behavior is shown by the following examples (which are necessarily more complicated than Ex. 6 and Ex. 7). First we need the following lemma.

Lemma 2. There exists a Banach space $C$, and a subspace $N$ of $C \times C$ with the following properties:

(i) $\pi_{1}(N)=\pi_{2}(N)=C$ (where $\pi_{1}$ (resp. $\pi_{2}$ ) denotes the projection on the first (resp. second) factor).

(ii) There exists an isomorphism $u$ (not necessarily norm preserving) from $N$ onto $C$.

(iii) There exists no continuous projection from $C \times C$ onto $N$.

Since the proof of this lemma would contribute very little additional insight into the subject matter of this paper, we omit it. We do, however, want to express our sincere thanks to W. F. Stinespring, $H$. Rubin, and especially to R. C. James, for contributing various steps in the construction of the spaces in Lemma 2. 
In the following examples, $C$ and $N$ are the spaces of Lemma 2.

Example 8. $E=N, F=G=C, K=\left\{\pi_{1}\left|N, \pi_{2}\right| N\right\}$. Then (1) does not hold, since $u \in \mathcal{L}(E, G)$ but $u \notin L$.

Example 9. $E=N, F=G=C, K=\left\{\pi_{1}\left|N, \pi_{2}\right| N, u\right\}$. Then (1) clearly holds. But (2) does not hold, which is seen by taking $\Im$ $=\left\{\pi_{1}\left|N, \pi_{2}\right| N\right\}$, and $V=u$.

\section{BIBLIOGRAPHY}

1. J. Dieudonne, La dualite dans les espaces vectoriels topologiques, Ann. Ecole Norm. (3) vol. 59 (1942) pp. 107-139.

2. J. Dixmier, Les fonctionelles linéaires sur l'ensemble des operrateurs bornés d'un espace de Hilbert, Ann. of Math. (2) vol. 51 (1950) pp. 387-408.

3. N. Jacobson, Structure theory of simple rings without finiteness assumptions, Trans. Amer. Math. Soc. vol. 57 (1945) pp. 228-245.

4. L. Nachbin, $A$ theorem of the Hahn-Banach type for linear transformations, Trans. Amer. Math. Soc. vol. 68 (1950) pp. 28-46.

5. R. S. Phillips, On linear transformation, Trans. Amer. Math. Soc. vol. 48 (1940) pp. 516-541.

6. C. E. Rickart, The uniqueness of norm problem in Banach algebras, Ann. of Math. (2) vol. 51 (1950) pp. 615-628.

7. B. Yood, Additive groups and linear manifolds of transformations between Banach spaces, Amer. J. Math. vol. 71 (1949) pp. 663-677.

University of Chicago 